\title{
Spin Correlation and Discrete Symmetry in Spinor Bose-Einstein Condensates
}

\author{
Fei Zhou \\ Physics Department, Princeton university, Princeton, NJ 08544, USA \\ ITP, Utrecht University, Princetonplein 5, 3584 CC Utrecht, The Netherlands*
}

\begin{abstract}
We study spin correlations in Bose-Einstein condensates of spin 1 bosons with scatterings dominated by a total spin equal 2 channel. We show that the low energy spin dynamics in the system can be mapped into an $o(n)$ nonlinear sigma model(NL $\sigma \mathrm{M}) . n=3$ at the zero magnetic field limit and $n=2$ in the presence of weak magnetic fields. In an ordered phase, the ground state has a discrete $Z_{2}$ symmetry and the degeneracy space is $\left[U(1) \times S^{n-1}\right] / Z_{2}$. We explore consequences of the discrete symmetry and propose some measurements to probe it.
\end{abstract}

Recently, there has been a burst of theoretical and experimental activities on spinor Bose-Einstein condensates [1] 6]. An optical trap confines alkali atoms independent of spins and liberates spin degree of freedoms [2.3. For sodium $\left({ }^{23} \mathrm{Na}\right)$ or rubidium $\left({ }^{87} \mathrm{Rb}\right)$ atoms with nuclear spin $I=3 / 2$ and electrons at $s$ orbits, the energy splitting between hyperfine multiplets is of order $100 \mathrm{mk}$. At temperatures as low as $100 \mathrm{nk},{ }^{23} \mathrm{Na}$ and ${ }^{87} \mathrm{Rb}$ atoms can be considered as simple bosons with a hyperfine spin $F=1$. The ground state of $\mathrm{N}$ spin 1 noninteracting bosons has $(N+1) N / 2$ folds spin degeneracy, by contrast to its magnetically trapped cousins. Presumably, hyperfine spin-dependent scattering lifts the degeneracy and leads to a spin correlated state. Optically trapped BEC therefore sets up a platform for studying quantum magnetism in many boson systems and adds a new dimension to already extremely rich physics in these systems.

The spin-dependent two -body interaction in BEC is characterized by $U_{2}\left(\mathbf{r}_{\mathbf{1}}, \mathbf{r}_{\mathbf{2}}\right)=\delta\left(\mathbf{r}_{1}-\mathbf{r}_{2}\right)\left[c_{0}+c_{2} \mathbf{F}_{\mathbf{1}} \dot{\mathbf{F}}_{\mathbf{2}}\right]$, as suggested in an early paper [⿶]. Here $c_{0}=\left(g_{0}+2 g_{2}\right) / 3$, $c_{2}=\left(g_{2}-g_{0}\right) / 3 ; g_{F}=4 \pi \hbar^{2} a_{F} / M, \mathrm{M}$ is the mass of the atom and $a_{F}$ is the s-wave scattering length in the total spin $F$ channel. Thus, the spin correlation in a BEC is determined by $c_{2}$. For ${ }^{87} R b, g_{2}<g_{0}$ or $c_{2}<0$ and the scattering is dominated by the total spin $F=0$ channel. In the ground state, all spins of atoms prefer to align in a certain direction and have a maxima magnetization [4].

For ${ }^{23} \mathrm{Na}$ studied experimentally [2], the scattering between ${ }^{23} \mathrm{Na}$ atoms is dominated by the total spin $F=2$ channel, i.e. $g_{2}>g_{0}$. The scattering between ${ }^{23} \mathrm{Na}$ atoms thus leads to an "antiferromagnetic" spin correlation. Efforts have been made to understand the ground state properties, exact excitation spectra, collec-

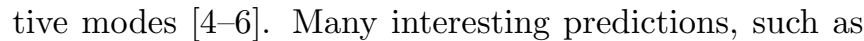
spin waves, spin mixing dynamics were made for ${ }^{23} \mathrm{Na}$ BEC where $c_{2}>0$.

In this paper, we show the spin dynamics in BEC with $c_{2}>0$ is characterized by an $o(n)$ nonlinear sigma model (NL $\sigma M)$ of $n$ components. $n=3$ at zero magnetic field limit and $n=2$ in the presence of a weak magnetic field. Spin correlations in spinor BEC can be studied in the context of the NL $\sigma \mathrm{M}$. We identify that the internal order parameter space for BEC as $\left[S^{1} \times S^{2}\right] / Z_{2}$ (zero field limit) and the ground state is nematically ordered. We explore consequences of the discrete $Z_{2}$ symmetry.

To describe the spin correlated BEC, it is most convenient to introduce Weyl representation of $S U(2)$ involving polynomials of a unit vector $(u, v)[7]$. Each unit vector is represented by a point $\Omega$ on a sphere with polar coordinates $(\theta, \phi)$; namely $u=\exp (i \phi / 2) \cos (\theta / 2)$, $v=\exp (-i \phi / 2) \sin \theta / 2$. The corresponding hyperfine spin operators are $F^{+}=u \partial / \partial v, F^{-}=v \partial / \partial u$, and $F_{z}=(u \partial / \partial u-v \partial / \partial v) / 2$. The scalar product between two wavefunctions $g$ and $f$ is defined as $\int g^{*}(u, v) f(u, v) d \Omega / 4 \pi$. (We reserve $\boldsymbol{\Omega}$ for the spin rotations discussed below.)

Under spin rotations $\mathcal{R}=\exp \left(i F_{z} \chi_{1} / 2\right) \exp \left(i F_{y} \theta_{1} / 2\right)$ $\exp \left(i F_{z} \phi_{1} / 2\right), u$ and $v$ transform into

$$
\begin{aligned}
& u\left(\boldsymbol{\Omega}_{\mathbf{1}}, \chi_{1}\right)=\exp \left(i \chi_{1} / 2\right) \\
& \left(\cos \theta_{1} / 2 \exp \left(-i \phi_{1} / 2\right) u+\sin \theta_{1} / 2 \exp \left(i \phi_{1} / 2\right) v\right), \\
& v\left(\boldsymbol{\Omega}_{\mathbf{2}}, \chi_{2}\right)=\exp \left(-i \chi_{2} / 2\right) \\
& \left(-\sin \theta_{2} / 2 \exp \left(-i \phi_{2} / 2\right) u+\cos \theta_{2} / 2 \exp \left(i \phi_{2} / 2\right) v\right),
\end{aligned}
$$

where $\boldsymbol{\Omega}_{1,2}=\left(\theta_{1,2}, \phi_{1,2}\right)$. Spin- 1 wavefunctions are polynomials of degree 2 in $u$ and $v \cdot \sqrt{3} u^{2}, \sqrt{6} u v, \sqrt{3} v^{2}$ correspond to $m=1,0,-1$ states. All $F=1$ states can also be expressed in term of $\sqrt{6} u\left(\boldsymbol{\Omega}_{\mathbf{1}}\right) v\left(\boldsymbol{\Omega}_{\mathbf{2}}\right)$ with $\boldsymbol{\Omega}_{1,2}$ properly chosen.

The Hamiltonian for spin-1 bosons can be written as

$$
\begin{aligned}
& \mathcal{H}=-\frac{1}{2 M} \sum_{\alpha} \nabla_{\alpha}^{2}+\sum_{\alpha, \beta}\left[\frac{c_{0}}{2}+\frac{c_{2}}{2} \mathbf{F}_{\alpha} \cdot \mathbf{F}_{\beta}\right] \delta\left(\mathbf{r}-\mathbf{r}^{\prime}\right) \\
& -\sum_{\alpha} \mathbf{F}_{z \alpha} g \mu_{B} H .
\end{aligned}
$$

${ }^{*}$ Permanent address 
The second term is hyperfine spin-dependent interaction with $c_{2}>0$ and the last term is the coupling with an external magnetic field $\mathbf{H}=H \mathbf{e}_{z} ; g$ is a Lande factor of an atom and $\mu_{B}$ is the Bohr magneton.

The wavefunction of $\mathrm{N}$ spin-1 Bosons generally can be written as 8

$\Psi\left(\left\{\mathbf{r}_{\alpha}\right\}\right)=\mathcal{P} \Pi_{\alpha=1 \ldots N} \Phi_{N_{\alpha}}\left(\mathbf{r}_{\alpha}\right) \sqrt{6} u_{\alpha}\left(\boldsymbol{\Omega}_{1 \alpha}\left(\mathbf{r}_{\alpha}\right)\right) v_{\alpha}\left(\boldsymbol{\Omega}_{2 \alpha}\left(\mathbf{r}_{\alpha}\right)\right)$.

$\mathcal{P}$ is the permutation of $\left\{N_{\alpha}\right\},\left\{\boldsymbol{\Omega}_{1 \alpha}, \boldsymbol{\Omega}_{2 \alpha}\right\} . N_{\alpha}$ labels an one-particle orbital state. Phases $\chi_{1,2}$ in Eq.1 are absorbed by a gauge transformation of the wavefunction $\Phi_{N_{\alpha}}$ introduced above. For BEC under consideration, we take $\boldsymbol{\Omega}_{1 \alpha, 2 \alpha}=\boldsymbol{\Omega}_{1,2}(\mathbf{r})$ and $\Phi_{N_{\alpha}}(\mathbf{r})=\Phi(\mathbf{r})(\Phi(\mathbf{r})$ is a complex scalar field). By introducing $\mathbf{n}(\mathbf{r})=\left(\boldsymbol{\Omega}_{1}+\boldsymbol{\Omega}_{2}\right) / 2$, $\mathbf{L}(\mathbf{r})=\left(\boldsymbol{\Omega}_{1}-\boldsymbol{\Omega}_{2}\right) / 2$ and $\Phi(\mathbf{r})=\sqrt{\rho(\mathbf{r})} \exp (i \chi(\mathbf{r}))$, we derive from Eqs.2,3 an effective Hamiltonian as a function of two pairs of variables : $\{\mathbf{n}(\mathbf{r}), \mathbf{L}(\mathbf{r})\}$ and $\{\rho(\mathbf{r}), \chi(\mathbf{r})\}$. These are collective variables of the $\mathrm{N}$ interacting spin1 Bosons, which describe the spin dynamics and phase dynamics respectively. In this representation, the hyperfine spin dependent interaction in Eq.2 is mapped into $\mathcal{H}_{s}=\int d \mathbf{r} c_{2} \mathbf{L}^{2}(\mathbf{r}) \rho^{2}(\mathbf{r})$, which only depends on collective variable $\mathbf{L}$ when $\rho(\mathbf{r})$ is taken as a constant. This indicates that each atom acquires an inertial $I_{0}=1 / 2 c_{2} \rho$ in the presence of hyperfine spin dependent scatterings with $c_{2}>0$; the rotation energy in the presence of a finite spin moment $\mathbf{L}$ is thus $\mathbf{L}^{2} / 2 I_{0}$.

In the most interesting limit, we can introduce the local spin density as $\mathbf{l}(\mathbf{r})=\mathbf{L}(\mathbf{r}) \rho(\mathbf{r})$. $\mathbf{n}$ and $\mathbf{l}$ satisfy the constrain $\mathbf{n}(\mathbf{r}) \cdot \mathbf{l}(\mathbf{r})=0$. Commutation relations between $\rho$ and $\chi, \mathbf{n}(\mathbf{r})$ and $\mathbf{l}(\mathbf{r})$ are given as $\left[\rho(\mathbf{r}), \chi\left(\mathbf{r}^{\prime}\right)\right]=i \hbar \delta(\mathbf{r}-$ $\left.\mathbf{r}^{\prime}\right) ;\left[\mathbf{n}_{\alpha}(\mathbf{r}), \mathbf{n}_{\beta}\left(\mathbf{r}^{\prime}\right)\right]=0,\left[\mathbf{l}_{\alpha}(\mathbf{r}), \mathbf{n}_{\beta}\left(\mathbf{r}^{\prime}\right)\right]=i \hbar \epsilon^{\alpha \beta \gamma} \mathbf{n}_{\gamma} \delta(\mathbf{r}-$ $\left.\mathbf{r}^{\prime}\right),\left[\mathbf{l}_{\alpha}(\mathbf{r}), \mathbf{l}_{\beta}\left(\mathbf{r}^{\prime}\right)\right]=i \hbar \epsilon^{\alpha \beta \gamma} \mathbf{l}_{\gamma} \delta\left(\mathbf{r}-\mathbf{r}^{\prime}\right) . \quad \epsilon^{\alpha \beta \gamma}$ is an antisymmetric tensor. These identities are valid when $\mathbf{L}$ per atom is much less than unity and $\mathbf{n}(\mathbf{r})$ can be considered as a classical "vector", components of which commutate with each other. The corresponding Lagrangian density can be derived as $\mathcal{L}=\mathcal{L}_{s}+\mathcal{L}_{c}+\mathcal{L}_{s c}$ [8], with

$$
\begin{aligned}
& \mathcal{L}_{c} \approx \frac{\rho}{2 M}\left[(\nabla \chi(\mathbf{r}))^{2}+\frac{1}{v_{c}^{2}}\left(\partial_{\tau} \chi\right)^{2}\right], \\
& \mathcal{L}_{s}=\frac{\rho}{2 M}\left[(\nabla \mathbf{n}(\mathbf{r}))^{2}+\frac{1}{v_{s}^{2}}\left(\partial_{\tau} \mathbf{n}\right)^{2}\right], \\
& \mathcal{L}_{c s}=\frac{\rho}{M} \frac{1}{v_{s}^{2}}\left[\mathbf{n} \times \partial_{\tau} \mathbf{n} \cdot \mathbf{n} \times(\nabla \chi \cdot \nabla) \mathbf{n}\right] .
\end{aligned}
$$

Here $\rho=\rho(0), v_{c}=\sqrt{2 \rho c_{0} / M}, v_{s}=\sqrt{2 \rho c_{2} / M}$. We introduce $\tau=$ it as the imaginary time. Nonlinearality is imposed via a constraint $\left|\mathbf{n}^{2}\right|=1$ at a low frequency limit. Eq.4 is the main result of the mapping and we have kept contributions which are lowest order in terms of $\partial_{\tau}$ and $\nabla . \mathcal{L}_{c}$ is taken in a Gaussian approximation and should be replaced by a full Gross-Pitaveskii Lagrangian in general. We will be mostly interested in the spin sector and simplification in $\mathcal{L}_{c}$ doesn't affect conclusions here. $\mathcal{L}_{s}$ in Eq.4 represents an $o(3) \mathrm{NL} \sigma \mathrm{M}$.

The last term $\mathcal{L}_{s c}$ characterizes a coupling between a spin rotation and the superflow in BEC due to Berry's phase. This term, however, is linear in $\partial_{\tau}$ and quadratic in spatial gradient and is negligible compared with $\mathcal{L}_{s}, \mathcal{L}_{c}$ at the long wave length limit. Particularly, such a coupling vanishes in a configuration where $\mathbf{L}$ is zero.

At the zero field limit, there exists a saddle point solution for the spin sector $\mathbf{n}(\mathbf{r})=\mathbf{n}_{0}, \mathbf{l}(\mathbf{r})=0$. $\mathbf{n}_{0}$ lives on a unit sphere. By expanding Eq.4 around the saddle point solution, we obtain spin waves with sound like spectrum $\omega=\sqrt{4 c_{2} \rho / M} k$, which can also be obtained in the GrossPitaveskii approach [4]. However, Eq.4 here is valid for any point $\boldsymbol{\Omega}_{1} \sim \boldsymbol{\Omega}_{2}$ on the unit sphere. The effective NL $\sigma \mathrm{M}$ derived here allows us to describe spin correlated states well beyond the Gross-Pitaveskii approach.

For a symmetry broken state, the ground state wavefunction in Eq.3 with $\boldsymbol{\Omega}_{1}=\boldsymbol{\Omega}_{2}=\mathbf{n}$ is invariant under a global transformation $\mathbf{n}, \chi \rightarrow-\mathbf{n}, \chi+\pi$;

$$
\Psi(\mathbf{n}, \chi)=\Psi(-\mathbf{n}, \chi+\pi), \Psi(\mathbf{n})=(-1)^{N} \Psi(-\mathbf{n})
$$

where $\chi$ is the phase of the scalar field $\Phi(x)$ introduced before. In obtaining this symmetry, we notice $u(\mathbf{n})=\exp (i \pi / 2) v(-\mathbf{n})$, with $\pi / 2$ from a phase of a spin$1 / 2$ particle under a $180^{\circ}$ rotation. Eq. 5 shows that the many-body wavefunctions characterized by $(\mathbf{n}, \chi)$ and $(-\mathbf{n}, \chi+\pi)$ are indistinguishable. Thus, the spinor BEC under considerations has a discrete $Z_{2}$ symmetry and the order parameter space is $\mathcal{R}=\left[S^{1} \times S^{2}\right] / Z_{2}$, with $Z_{2}$ as a two-element group of integers modulo 2 .

As in a classical uniaxial nematic liquid crystal where diatomic molecules are indistinguishable upon an inversion of their directors $\mathbf{n}$ and the internal order parameter space is $S^{2} / Z_{2}$, the $Z_{2}$ symmetry identified here also indicates that there exists a tensor order parameter [9]. For the purpose of demonstration, let us introduce a "director" $\mathbf{d}_{x}=\left[u v^{*}+v u^{*}\right] / 2, \mathbf{d}_{y}=\left[u v^{*}-v u^{*}\right] / 2 i$, $\mathbf{d}_{z}=\left[u u^{*}-v v^{*}\right] / 2$. One then can show for the ground state wavefunction in Eq. $3,<\mathbf{d}>=0$ but nematic order parameter $Q_{\alpha \beta}=1 / \rho\left[<\mathbf{d}_{\alpha} \mathbf{d}_{\beta}>-1 / 3 T r<\mathbf{d}_{\alpha} \mathbf{d}_{\beta}>\right]$ is nonzero. Here $\langle>$ stands for an average taken over the ground state wave function $\Psi$. In fact,

$$
Q_{\alpha \beta}=\frac{2}{5}\left[-3 \mathbf{n}_{0 \alpha} \mathbf{n}_{0 \beta}+\delta_{\alpha \beta}\right]
$$

According to Eq.6, the director $\mathbf{d}$ aligns in a plane perpendicular to $\mathbf{n}_{0}$.

Topological defects in this case are of particular interest because of the discrete symmetry in Eq.5. Following the general theory for the classification of defects in a symmetry broken state, the $Z_{2}$ symmetry leads to $\pi$ spin disclinations superimposed with half vortices, which otherwise don't exist. The corresponding wavefunction of composite linear singularities $\left(Z_{2}\right.$ strings $)$ is 


$$
\begin{aligned}
& \lim _{\xi \rightarrow \infty} \mathbf{n}(\xi)=\operatorname{Re}\left(\frac{\xi-\xi_{0}}{\left|\xi-\xi_{0}\right|}\right)^{m+1 / 2} \mathbf{e}_{x}+\operatorname{Im}\left(\frac{\xi-\xi_{0}}{\left|\xi-\xi_{0}\right|}\right)^{m+1 / 2} \mathbf{e}_{y} \\
& \lim _{\xi \rightarrow \infty} \mathbf{v}_{s}(\xi)=\frac{1 / 2+n}{M\left|\xi-\xi_{0}\right|} \\
& {\left[\operatorname{Im}\left(\frac{\xi-\xi_{0}}{\left|\xi-\xi_{0}\right|}\right) \mathbf{e}_{x}-\operatorname{Re}\left(\frac{\xi-\xi_{0}}{\left|\xi-\xi_{0}\right|}\right) \mathbf{e}_{y}\right] .}
\end{aligned}
$$

Here $\xi=x+i y$ and lines are located at $\xi_{0}=x_{0}+i y_{0} ; n, m$ are integers and $\mathbf{v}_{s}$ is superfluid velocity [10]. Each string is characterized by $(m, n)$. However, $(-1, n),( \pm 3, n)$, $( \pm 5, n)$ strings can be obtained by deforming string $(1, n)$ and are homotopical identical to $(1, n)$.

In a composite string given in Eq.7, $\mathbf{n}$ evolves into $-\mathbf{n}$ along a loop enclosing $\xi_{0}$. The corresponding spin wavefunction changes its sign under an inversion $\mathbf{n} \rightarrow-\mathbf{n}$, following the identity $u(\mathbf{n}) v(\mathbf{n})=-u(-\mathbf{n}) v(-\mathbf{n})$. A superflow $\mathbf{v}_{s}$ of a half vortex is present to compensate the $\pi$ phase under $\mathbf{n} \rightarrow-\mathbf{n}$ rotation and ensure the single valuedness of the wavefunction. This composite structure is different from a linear defect in a classical nematic liquid where $\pi$ disclinations are free topological excitations. In fact, in a coherent spinor BEC, a bare $\pi$-spin disclination carries a cut along which phase changes abruptly from $\pi$ to $2 \pi$ or 0 . This cut starting at the disclination ends only at the boundary of the system and costs an energy linear in term of the size of BEC. For a similar reason, the energy cost to have a $\pi$ disclination and a half-vortex separated at a distance $L$ is linearly proportional to $L$. Composite strings in Eq.7 should be considered as results of confinement of $\pi$ spin disclinations and half vortices in spinor BEC [1]].

In the presence of an external magnetic field along $z$ direction, $\boldsymbol{\Omega}_{1,2}=\mathbf{n} \pm \mathbf{e}_{z} g \mu_{B} H / 4 c_{2} \rho$ and $\mathbf{n}$ satisfies constrain

$$
\mathbf{n} \cdot \frac{\mu_{B} H}{4 c_{2} \rho} \mathbf{e}_{z}=0 .
$$

Obviously, an external magnetic field breaks the $S^{2}$ symmetry and confines low frequency sector of $\mathbf{n}$ in a plane perpendicular to $\mathbf{H}$ itself. The Lagrangian in the presence of a magnetic field is that of an $O(2) \mathrm{NL} \sigma \mathrm{M}$; it has $S^{1}$-symmetry at the frequency $\omega \ll \mu_{B} H$. At the high frequency limit, $\mathbf{n}$ precesses in a field $4 c_{2} \mathbf{l}(x)$, much larger than the external field and $S^{2}$-symmetry is restored.

As a consequence, the order parameter space for the quantum spin nematic state in an external magnetic field is $\mathcal{R}=\left[S^{1} \times S^{1}\right] / Z_{2}$. The nematic order parameter $Q_{\alpha \beta}$ is still given by Eq.7, with the easy plane of $\mathbf{d}$ parallel to the external field. Wavefunctions for linear defects are of same forms as those in Eq.7, but with all strings $(m, n)$ homotopically distinguishable.

We have restricted ourselves to the weak magnetic field limit and neglected the possible quadratic Zeemann shift $\mathcal{H}_{Q Z}=\sum_{\alpha} Q H^{2} F_{z \alpha}^{2}$ (the external field $H$ is along $\mathbf{e}_{z}$ direction). Inclusion of the quadratic Zeemann shift yields an additional term $\mathcal{L}_{Q Z}=\int \rho Q H^{2}\left(\mathbf{n}_{x}^{2}+\mathbf{n}_{y}^{2}\right) d x$ to the
$\mathrm{NL} \sigma \mathrm{M}$ derived. The main effect of this contribution is to align $\mathbf{n}$ along the external field. When this shift dominates, the ground state is left with a double degeneracy: $\mathbf{n}=\mathbf{e}_{z}$ and $\mathbf{n}=-\mathbf{e}_{z}$. The spin wave develops an energy gap of order $Q H^{2}$. We will focus on the zero magnetic field case in the rest of discussions.

In general, following Eq.4, spin correlated BEC can be studied by considering a NL $\sigma \mathrm{M}$,

$$
\begin{aligned}
& \mathcal{L}_{s}=\frac{1}{2 f}\left(\partial_{\mu} \mathbf{n}\right)^{2}, \mathbf{n}^{2}=1 ; \\
& f=(16 \pi)^{1 / 2}\left(\rho \Delta a^{3}\right)^{1 / 6}, \Delta a=\frac{a_{2}-a_{0}}{3} .
\end{aligned}
$$

We introduce dimensionless length and time: $\tilde{\mathbf{r}}=$ $\mathbf{r} \rho^{1 / 3}, \tilde{\tau}=\tau v_{s} \rho^{1 / 3}$. Derivatives $\partial_{\mu}$ are defined as $\left(\partial_{\tilde{\tau}}, \partial_{\tilde{x}}, \partial_{\tilde{y}}, \partial_{\tilde{z}}\right) . f^{-1}$ is a square root of the ratio between potential energy at an interatomic scale $\hbar^{2} \rho^{2 / 3} / 2 m$ and zero point kinetic(rotation) energy $c_{2} \rho / 2$ of an individual atom. The zero point motion is absent for noninteracting bosons but gets stronger when $c_{2}$ increases, or the effective inertial $I_{0}$ gets smaller. The $o(3) \mathrm{NL} \sigma \mathrm{M}$ has order and disordered phases at $d>1$, depending on the parameter $f$. Most of qualitative results about spin correlations in BEC can be obtained in a renormalization group (RG) approach [8]. For $\rho \Delta a^{3}=10^{-6}$ as is in experiments, a long range nematic order should be observed.

In $1 D$ case, at zero temperature and zero field, one should expect there will be no long range order and the state is nematically disordered, following the RG results of NL $\sigma \mathrm{M}$ [13]. These nematic disordered states mimic the quantum spin liquid states proposed in the literature of Heisenberg antiferromagnetic systems(HAFS). However, we notice that the NL $\sigma \mathrm{M}$ derived from the microscopic Hamiltonian in this paper doesn't have a $\theta$-term $\mathcal{L}_{\theta}=\theta / 4 \pi \int d \tau d x \mathbf{n} \cdot \partial \mathbf{n} / \partial \tau \times \partial \mathbf{n} / \partial x$, which is generally present in HAFS studied before [12]. Absence of a $\theta$-term, which ensures an energy gap in the excitation spectrum of nematic state, follows a fact that the Berry's phase under rotations of $\mathbf{n}$ vanishes identically [8].

Two remarks are in order. 1) At a high density limit, one should also take into three-body, four-body elastic scatterings. This can further modify the short distance dynamics but will not affect conclusions arrived above in a qualitative way. 2)One should be cautious about the definition of "phase" since the alkali atoms under investigation are in a long lived metal stable gaseous state. The life time of alkali atomic gas is limited by three-body inelastic collisions [14]. The collision rate is proportional to the square of the number density of atoms and increases dramatically as the density increases. This sets a practical limit in order for the quantum disordered nematic liquid to be probed in BEC.

Since the experiment [2] was done in a BEC cloud with a few millions sodium atoms, it is also particularly interesting to consider the symmetry restoring due to a finite size effect. We take a weakly interacting limit where 
quantum fluctuations of finite wave length are negligible. In a zero mode approximation, Hamiltonian becomes,

$$
\mathcal{H}_{z . m}=\rho c_{2} \frac{\mathbf{L}^{2}}{2 N}
$$

$\mathbf{L}$ is the total spin of the N-Bosons system and can be considered as an angular momentum operator defined on a unit sphere of $\mathbf{n}[8]$. And $[\mathbf{n}, \mathbf{n}]=0,\left[\mathbf{L}_{\alpha}, \mathbf{n}_{\beta}\right]=i \hbar \epsilon^{\alpha \beta \gamma} \mathbf{n}_{\gamma}$, $\left[\mathbf{L}_{\alpha}, \mathbf{L}_{\beta}\right]=i \hbar \epsilon^{\alpha \beta \gamma} \mathbf{L}_{\gamma}$. The energy spectrum is given by $\mathbf{L}^{2}=l(l+1)$, which is identical to that obtained in [6]. $l=0,2,4, \ldots . N$, if $N$ is an even number; and $l=1,3,5, \ldots$ otherwise. The energy gap between lowest lying excitations is inversely proportional of $N$ and vanishes at large $N$ limit.

Now consider a wave packet with $\mathbf{n}$ confined within a region centered at $\mathbf{n}_{0}=\mathbf{e}_{z}$ of size $\sqrt{\left\langle\delta^{2} \mathbf{n}\right\rangle_{0}} \ll 1$ on the unit sphere at $t=0$. Spread $\left\langle\delta^{2} \mathbf{n}\right\rangle$ is defined as the expectation value of $\mathbf{n}_{x}^{2}+\mathbf{n}_{y}^{2}$. In spherical polar coordinates $(\theta, \phi)$ where $\mathbf{n}$ is a vector represented by $(\sin \theta \cos \phi, \sin \theta \sin \phi, \cos \theta)$, the corresponding wave packet(for an even $N$ ) can be constructed as

$$
\Psi(\theta, \phi, t)=\frac{1}{B} \sum_{l=2 n} \exp \left(-\frac{l^{2}}{4 \sigma}-i t \frac{l(l+1) c_{2} \rho}{2 N}\right) Y_{l, 0}(\theta, \phi) .
$$

Here $B=\sum_{l} \exp \left(-l^{2} / 2 \sigma\right) ;\left\langle\delta^{2} \mathbf{n}>_{0}=A_{0} / \sigma\right.$ with constant $A_{0}$ estimated to be a constant of unity. $Y_{l, 0}(\theta, \phi)$ are spheric harmonics. $\sigma \gg 1$ but $\sigma / N$ is vanishingly small. The energy of this wave packet is $\Delta E=$ $2 A_{0} \sigma c_{2} \rho / N$. In the limit $N \rightarrow \infty$, an infinitesimal external field will stablize this wave packet with respect to the rotation invariant state.

The spread of $\mathbf{n}$ at a time $t$ is

$$
<(\delta \mathbf{n})^{2}>_{t}=<(\delta \mathbf{n})^{2}>_{0}+4 A_{0} \sigma\left(t \frac{c_{2} \rho}{N}\right)^{2},
$$

which is valid at $t<\tau_{c}=\sigma / A_{0} \Delta E$. At $t>\tau_{c}$, the spread is of order unity. Therefore, $\sqrt{\left\langle\delta^{2} \mathbf{n}\right\rangle_{t}}$ exceeds the initial spread $\sqrt{\left\langle\delta^{2} \mathbf{n}\right\rangle_{0}}$ at a time of order $1 / \Delta E$. At a longer time $\tau_{c}$, $\mathbf{n}$ starts to explore the whole unit sphere $S^{2}$ and the rotation symmetry is restored due to spin-dependent scatterings in BEC.

Eq.12 also imposes restrictions on measurements. A measurement of $\mathbf{n}$ in ${ }^{23} \mathrm{Na}$ BEC discussed here excites the ground state to an excited state of energy $\Delta E$, where $\mathbf{n}$ has a finite spread on $S^{2}$ and $\Delta E$ is infinitesimal small in the thermal dynamical limit. The time scale to restore the broken symmetry is determined by the twobody spin dependent scattering lengths and the number of atoms in BEC. A measurement taken at a time scale longer than $\sigma / A_{0} \Delta E$ should reveal the symmetry restoring because of zero point motions of $\mathbf{n}$. With $N \sim 10^{7}$ and $c_{2} \rho \sim 100 \mathrm{~Hz}(500 \mathrm{nK})$, the symmetry restoring time is about one day which is much longer than the life time of the trap. However, for a smaller cloud with $10^{3-4}$ atoms, the symmetry restoring can take place within $10-100$ secs, before recombination processes take place.

Nematically ordered BEC has very fascinating optical properties [8]. In the presence of spin-orbital couplings, dielectric constant is a tensor expressed in terms of $Q_{\alpha \beta}$. This suggests that birefringence takes place in the system as a direct evidence of the hidden $Z_{2}$ symmetry. Another experiment consequence associated with the broken symmetry is the enhanced small angle light scattering due to thermal fluctuations of $\mathbf{n}$. For ${ }^{23} \mathrm{Na}$, the light scattering amplitude can be four order of magnitude higher than that in an isotropic BEC and the nematic BEC is optically turbid. Finally, the $Z_{2}$ symmetry also implies that there exists a local $Z_{2}$ gauge field in BEC. This was recently considered and will be published elsewhere [15.

FZ would like to thank E. Demler, J.Ho for many stimulating discussions. He is particularly grateful to F. D. Haldane for his guidance and interest on this subject. This work was partially supported by ARO under DAAG 55-98-1-0270.

[1] C. J. Myatt et al., Phys. Rev. Lett. 78, 586(1997).

[2] D. M. Stamper-Kurn et al., Phys. Rev. Lett. 80, 2027(1998).

[3] J. Stenger et al., Nature 396, 345(1998).

[4] T. L. Ho, Phys. Rev. Lett. 81, 742 (1998); T. L. Ho and S. K. Yip, Phys. Rev. Lett. 84, 4031(2000).

[5] T. Ohmi and K. Machinda, J. Phys. Soc. Jpn. 67, 1822(1998).

[6] C. K. Law et al., Phys. Rev. Lett. 81, 5257(1998).

[7] D. P. Arovas et al., Phys. Rev. Lett. 60, 531(1988);I. Afflect, J. Phys. Condensed Matter 1, 3047(1989).

[8] F. Zhou and F.D. Haldane, Preprint ITP-UU$00 / 51(2000)$.

[9] P. G. de Gennes, The physics of liquid crystals, Oxford University Press(1974).

[10] Half vortices were also discussed by V. Leonhart and G. E. Volovik, JETP Lett. 72,46(2000).

[11] The vortex core is ferromagnetic with spins oriented along the direction of the flux, Haldane, unpublished.

[12] F. D. M. Haldane, Phys. Rev. Lett. 50, 1153(1983).

[13] A. M. Polyakov Gauge Fields and Strings, Hardwood academic publishers(1987).

[14] W. Ketterle, D. S. Durfee and D. M. Stamper-Kurn, in Bose-Einstein condensation in atomic gases, Proceedings of the international School of Physics Enrico Fermi, Course $C X L$, edited by M. Inguscio, S. Stringari and C. E. Wieman (IOS Press, Amsterdam, 1999).

[15] E.Demler and F.Zhou, cond-mat/0104409; E. Demler, F.Zhou and F.D. Haldane, Preprint ITP-UU01/09(2001). 